\title{
Controllability of nonautonomous boundary integrodifferential Cauchy problems
}

\author{
Mohammed Moussi
}




\title{
CONTROLLABILITY OF NONAUTONOMOUS BOUNDARY INTEGRODIFFERENTIAL CAUCHY PROBLEMS
}

\author{
M. MOUSSI \\ Received 05 December, 2013
}

\begin{abstract}
In this paper, we show the existence of mild solutions to nonautonomous boundary integrodifferential Cauchy problems. Moreover, we establish a controllability result. An example illustrates the obtained results.
\end{abstract}

2010 Mathematics Subject Classification: 34K10; 45K05; 93B05

Keywords: boundary exact controllability, nonautonomous boundary integrodifferential equations, evolution families, population equations

\section{INTRODUCTION}

We consider in the present paper the following nonlinear boundary integrodifferential Cauchy problem:

$$
\left\{\begin{array}{l}
\frac{d}{d t} x(t)=A_{\max }(t) x(t), \quad 0 \leq \tau \leq t \leq T, \\
L(t) x(t)=f\left(t, x(t), \int_{\tau}^{t} g(t, \sigma, x(\sigma)) d \sigma\right), \quad 0 \leq \tau \leq t \leq T, \\
x(\tau)=x_{0},
\end{array}\right.
$$

where $A_{\max }(t)$ are closed operators on a Banach space $X$ endowed with a maximal domain $D\left(A_{\max }(t)\right), L(t): D\left(A_{\max }(t)\right) \rightarrow \partial X$ with a 'boundary Banach space' $\partial X$, a function $f: \mathbb{R}_{+} \times X \times X \rightarrow \partial X$ and a function $g: \mathbb{R}_{+} \times \mathbb{R}_{+} \times X \rightarrow X$.

In recent years, such abstract integrodifferential equations have attracted the interest of many authors. Questions as existence of solutions, perturbations, controllability and the asymptotic behavior are the subject of many works. We cite among others $[1,3,4,6-8,10-12,14-17,20-23]$ and the references therein.

Our aim is to study the wellposedness of (1.1) and to present a controllability result.

In [2] we have studied the boundary Cauchy problem in the case that the second equation in (1.1) is replaced by an equation $L(t) x(t)=f(t)$. For this type of equation we established mild solutions given by a variation of constants formula. In this 
work, we firstly extend this to a variation of constants formula for problem (1.1) using the contraction fixed point theorem, see Section 2.

In Section 3 we present sufficient conditions to obtain exact controllability of the following boundary integrodifferential control system:

$$
\left\{\begin{array}{l}
\frac{d}{d t} x(t)=A_{\max }(t) x(t), \quad t \in[0, T], \\
L(t) x(t)=f\left(t, x(t), \int_{\tau}^{t} g(t, \sigma, x(\sigma)) d \sigma\right)+B(t) u(t) \quad t \in[0, T], \\
x(0)=x_{0} .
\end{array}\right.
$$

Here the input function $u(\cdot)$ takes values in a Banach space $U$ (the control space) and the nonautonomous control operator $B(t), t \in[0, T]$, are bounded from $U$ into $\partial X$.

To illustrate these assumptions and our results, we present in Section 4 a population equation as an example.

\section{MiLD SOLUTION OF NONLINEAR BOUNDARY INTEGRODIFFERENTIAL$$
\text { EQUATION }
$$

Consider the following nonlinear integrodifferential boundary Cauchy problem

$$
\left\{\begin{array}{l}
\frac{d}{d t} x(t)=A_{\max }(t) x(t), \quad 0 \leq \tau \leq t \leq T, \\
L(t) x(t)=f\left(t, x(t), \int_{\tau}^{t} g(t, \sigma, x(\sigma)) d \sigma\right), \quad \tau \leq t \leq T, \\
x(\tau)=x_{0} \in X
\end{array}\right.
$$

where $X$ is a Banach space, the operators $A_{\max }(t) \in \mathscr{L}(D, X)$ and $L(t) \in \mathscr{L}(D, \partial X)$ for $t \geq 0$, with $D$ and $\partial X$ Banach spaces such that $D$ is dense and continuously embedded in $X$.

The operators $A_{\max }(t)$ and $L(t)$ are supposed to satisfy the following hypotheses:

(H1) There are positive constants $C_{1}, C_{2}$ such that

$$
C_{1}\|x\|_{D} \leq\|x\|+\left\|A_{\max }(t) x\right\| \leq C_{2}\|x\|_{D}
$$

for all $x \in D$ and $t \geq 0$;

(H2) for each $x \in D$, the mapping $\mathbb{R}_{+} \ni t \mapsto A_{\max }(t) x \in X$ is continuously differentiable;

(H3) the operators $L(t): D \rightarrow \partial X, t \geq 0$, are surjective;

(H4) for each $x \in D$, the mapping $\mathbb{R}_{+} \ni t \mapsto L(t) x \in \partial X$ is continuously differentiable;

(H5) there exist constants $\gamma>0$ and $\omega \in \mathbb{R}$ such that

$$
\begin{aligned}
&\|L(t) x\|_{\partial X} \geq \gamma^{-1}(\lambda-\omega)\|x\|_{X}, \\
& \text { for } x \in \operatorname{ker}\left(\lambda-A_{\max }(t)\right), \lambda>\omega \text { and } t \geq 0 ;
\end{aligned}
$$


(H6) the family of operators $(A(t))_{0 \leq t \leq T}$, with $A(t):=\left.A_{\max }(t)\right|_{\operatorname{ker} L(t)}$, is stable, that is, there are constants $M \geq 1$ and $\omega \in \mathbb{R}$ such that $(\omega, \infty) \subset \rho(A(t))$ (the resolvent set of $A(t)) \forall 0 \leq t \leq T$ and

$$
\left\|\prod_{i=1}^{k} R\left(\lambda, A\left(t_{i}\right)\right)\right\| \leq M(\lambda-\omega)^{-k}
$$

for $\lambda>\omega$ and any finite sequence $0 \leq t_{1} \leq \cdots \leq t_{k} \leq T$, and $R(\lambda, A(t)):=(\lambda-A(t))^{-1}$.

In the following lemma we cite consequences of the above assumptions from [9, Lemma 1.2] which will be needed below.

Lemma 1. The restriction $\left.L(t)\right|_{\operatorname{ker}\left(\lambda-A_{\max }(t)\right)}$ is an isomorphism from $\operatorname{ker}\left(\lambda-A_{\max }(t)\right)$ into $\partial X$ and its inverse $L_{\lambda, t}:=\left[\left.L(t)\right|_{\operatorname{ker}\left(\lambda-A_{\max }(t)\right)}\right]^{-1}: \partial X \rightarrow$ $\operatorname{ker}\left(\lambda-A_{\max }(t)\right)$ satisfies

$$
\left\|L_{\lambda, t}\right\| \leq \gamma(\lambda-\omega)^{-1} \quad \text { for } \lambda>\omega .
$$

Recall that in case $f \equiv 0$ the problem (2.1) is reduced to the linear boundary Cauchy problem

$$
\left\{\begin{array}{l}
\frac{d}{d t} x(t)=A_{\max }(t) x(t), \quad \tau \leq t \leq T, \\
L(t) x(t)=0, \quad \tau \leq t \leq T, \\
x(\tau)=x_{0}
\end{array}\right.
$$

which was studied by Kellermann [13] and Nguyen Lan [18]. In particular, the authors proved that under assumptions (H1)-(H6) the problem (2.2) has a unique solution given by an evolution family $(U(t, \tau))_{\tau \leq t \leq T}$ satisfying

$$
\|U(t, \tau)\| \leq M e^{\omega(t-\tau)}, \quad \forall \tau \leq t \leq T,
$$

where $M$ and $\omega$ are the stability constants of $A(t)$.

In this section we study the existence of mild solutions of the nonlinear problem (2.1) and assume for the nonlinear part:

(H7) The nonlinear function $f:[0, T] \times X \times X \rightarrow \partial X$ is continuous and there exists a positive constant $\ell_{f}$ such that one has the following global Lipschitz estimate

$$
\|f(t, x, y)-f(t, \bar{x}, \bar{y})\| \leq \ell_{f}(\|x-\bar{x}\|+\|y-\bar{y}\|)
$$

for all $x, y, \bar{x}, \bar{y} \in X$ and $t \in[0, T]$;

(H8) the nonlinear function $g: \Delta_{T} \times X \rightarrow X$ is continuous and there exists a positive constant $\ell_{g}$ such that one has the global Lipschitz estimate

$$
\|g(t, s, x)-g(t, s, \bar{x})\| \leq \ell_{g}\|x-\bar{x}\|
$$

for all $x, \bar{x} \in X$ and $(t, s) \in \Delta_{T}$. Here $\Delta_{T}$ is defined by:

$$
\Delta_{T}:=\{(t, s): 0 \leq s \leq t \leq T\} .
$$


Under the assumptions (H1)-(H8) and using the usual contraction argument we shall show the existence of a unique mild solution given by the following definition.

Definition 1. A continuous function $x(\cdot):[\tau, T] \rightarrow X$ is called mild solution of the problem (2.1) if it satisfies the variation of constants formula

$$
x(t)=U(t, \tau) x_{0}+\lim _{\lambda \rightarrow \infty} \int_{\tau}^{t} U(t, \sigma) \lambda L_{\lambda} f\left(\sigma, x(\sigma), \int_{\tau}^{\sigma} g(\sigma, \alpha, x(\alpha)) d \alpha\right) d \sigma
$$

for all $\tau \leq t \leq T$.

Remark 1. The limit in equation (2.4) is well-defined, for details we refer the reader to [2].

In the following theorem we prove the existence of a mild solution.

Theorem 1. Let the assumptions (H1)-(H8) be satisfied. Then for every $x_{0} \in X$ and $\tau \geq 0$ the boundary integrodifferential equation (2.1) has a unique mild solution on $[\tau, T]$.

Proof. Let $x_{0} \in X$ be fixed. Let $Y:=C([0, T], X)$ be the Banach space of all continuous functions from $[0, T]$ into $X$. Define an operator

$(\Phi v)(t):=U(t, \tau) x_{0}+\lim _{\lambda \rightarrow \infty} \int_{\tau}^{t} U(t, \sigma) \lambda L_{\lambda, \sigma} f\left(\sigma, v(\sigma), \int_{\tau}^{\sigma} g(\sigma, \alpha, v(\alpha)) d \alpha\right) d \sigma$

for all $\tau \leq t \leq T$ and $v \in Y$. It is clear that $\Phi$ maps $Y$ into itself.

Now for $v_{1}, v_{2}$ in $Y$ we have

$$
\begin{aligned}
\left\|\left(\Phi v_{1}\right)(t)-\left(\Phi v_{2}\right)(t)\right\| & \\
\leq & \| \lim _{\lambda \rightarrow \infty} \int_{\tau}^{t} U(t, \sigma) \lambda L_{\lambda, \sigma} f\left(\sigma, v_{1}(\sigma), \int_{\tau}^{\sigma} g\left(\sigma, \alpha, v_{1}(\alpha)\right) d \alpha\right) \\
& -f\left(\sigma, v_{2}(\sigma), \int_{\tau}^{\sigma} g\left(\sigma, \alpha, v_{2}(\alpha)\right) d \alpha\right) d \sigma \| .
\end{aligned}
$$

Using (2.3), assumption (H7) and assumption (H8) we obtain

$$
\begin{aligned}
\left\|\left(\Phi v_{1}\right)(t)-\left(\Phi v_{2}\right)(t)\right\| \leq & \int_{\tau}^{t} \gamma M e^{\omega(t-\sigma)} \ell_{f}\left(\left\|v_{1}(\sigma)-v_{2}(\sigma)\right\|\right. \\
& \left.+\left\|\int_{\tau}^{\sigma} g\left(\sigma, \alpha, v_{1}(\alpha)\right)-g\left(\sigma, \alpha, v_{2}(\alpha)\right) d \alpha\right\|\right) d \sigma \\
\leq & \int_{\tau}^{t} \gamma M e^{\omega(t-\sigma)} \ell_{f}\left(\left\|v_{1}-v_{2}\right\|+\ell_{g}(\sigma-\tau)\left\|v_{1}-v_{2}\right\|\right) d \sigma \\
\leq & e^{\omega(t-\tau)} \gamma M(t-\tau) \ell_{f}\left(\left\|v_{1}-v_{2}\right\|+\ell_{g}(t-\tau)\left\|v_{1}-v_{2}\right\|\right) \\
\leq & e^{\omega(t-\tau)} \gamma M \ell_{f}(t-\tau)\left(1+\ell_{g} T\right)\left\|v_{1}-v_{2}\right\| .
\end{aligned}
$$




$$
\leq e^{\omega T} \gamma M \ell_{f} T\left(1+\ell_{g} T\right)\left\|v_{1}-v_{2}\right\| .
$$

By induction, one obtains

$$
\left\|\Phi^{n} v_{1}-\Phi^{n} v_{2}\right\| \leq e^{\omega T} \frac{\left(\gamma M \ell_{f} T\left(1+\ell_{g} T\right)\right)^{n}}{n !}\left\|v_{1}-v_{2}\right\|
$$

which implies that, for $n$ sufficiently large, $\Phi^{n}$ is a contraction map on the Banach space $Y$. Hence by the Banach fixed point theorem there exists a unique function $v \in Y$ satisfying $\Phi v=v$ which is the mild solution of the problem (2.1).

\section{BOUNDARY INTERGRODIFFERENTIAL CONTROLLED SYSTEM}

In this section we consider the following boundary control system

$$
\left\{\begin{array}{l}
\frac{d}{d t} x(t)=A_{\max }(t) x(t), \quad t \in[0, T], \\
L(t) x(t)=f\left(t, x(t), \int_{0}^{t} g(t, \sigma, x(\sigma)) d \sigma\right)+B(t) u(t) \quad t \in[0, T], \\
x(0)=x_{0} .
\end{array}\right.
$$

Here $A_{\max }(t), L(t), f(t, \cdot, \cdot), g(t, \cdot, \cdot)$ satisfy the hypotheses $(\mathrm{H} 1)-\mathrm{H}(8)$, the control function $u(\cdot)$ takes values in a Banach space $U$ and $B(t)$ are bounded operators from $U$ into $\partial X$. As in the previous section, one can show that the control system (3.1) admits a unique mild solution $x(\cdot)$ given by

$$
\begin{aligned}
x(t)= & U(t, 0) x_{0}+\lim _{\lambda \rightarrow \infty} \int_{0}^{t} U(t, \sigma) \lambda L_{\lambda} \\
& \times\left[f\left(\sigma, x(\sigma), \int_{0}^{\sigma} g(\sigma, \alpha, x(\alpha)) d \alpha\right)+B(\sigma) u(\sigma)\right] d \sigma
\end{aligned}
$$

for $t \geq 0$ and $x_{0} \in X$.

Our aim in this section is to present sufficient conditions to obtain the exact controllability of the controlled system (3.1). We first recall the following definition.

Definition 2. The control system (3.1) is said to be exactly controllable on the interval $[0, T]$ for some $T>0$, if for all $x_{0}, y \in X$, there exists a control $u \in$ $L^{2}([0, T], U)$ such that the mild solution $x(t)$ of (3.1) corresponding to $u$ satisfies $x(T)=y$.

In order to reach the goal of this section, we further assume the following additional hypothesis.

(H9) The operator $W: L^{2}([0, T], U) \longrightarrow X$ defined by

$$
W u:=\lim _{\lambda \rightarrow \infty} \int_{0}^{T} U(t, \sigma) \lambda L_{\lambda} B(\sigma) u(\sigma) d \sigma
$$


has a pseudo inverse operator $W^{-1}$ which takes values in $L^{2}([0, T], U) / \operatorname{ker}(W)$ and there exist positive constants $k_{1}, k_{2}$ such that

$$
\|B(\cdot)\| \leq k_{1} \text { and }\left\|W^{-1}\right\| \leq k_{2} .
$$

Here, $\operatorname{ker}(W)$ denotes the kernel of the operator $W$.

Remark 2. For the construction of $W$ and $W^{-1}$ we refer to the paper [19].

Our overall approach is the usual fixed point method. Therefore, define the operator $F: C([0, T], X) \longrightarrow C([0, T], X)$ by

$$
\begin{aligned}
(F x)(t):= & U(t, 0) x_{0}+\lim _{\lambda \rightarrow \infty} \int_{0}^{t} U(t, \sigma) \lambda L_{\lambda} \\
& \times\left[f\left(\sigma, x(\sigma), \int_{0}^{\sigma} g(\sigma, \alpha, x(\alpha)) d \alpha\right)+B(\sigma) u(\sigma)\right] d \sigma .
\end{aligned}
$$

Here the control $u(\cdot)$ is chosen for $x(\cdot) \in C([0, T], X)$ and $y \in X$ as

$$
\begin{aligned}
u(t):= & W^{-1}\left[y-U(T, 0) x_{0}-\lim _{\lambda \rightarrow \infty} \int_{0}^{T} U(t, \sigma) \lambda L_{\lambda}\right. \\
& \left.\times f\left(\sigma, x(\sigma), \int_{0}^{\sigma} g(\sigma, \alpha, x(\alpha)) d \alpha\right) d \sigma\right](t) .
\end{aligned}
$$

We have the following proposition.

Proposition 1. The mapping $F$ is a contraction from the Banach space $C([0, T], X)$ into itself provided that $\left(1+T \ell_{g}\right)\left(\gamma M T \ell_{f}+\gamma^{2} M^{2} k_{1} k_{2} \ell_{f} T\right)<1$.

Proof. Let $x_{1}, x_{2} \in C([0, T], X)$ and choose control functions $u_{1}, u_{2}$ respectively. We have

$$
\begin{gathered}
\left(F x_{1}\right)(t)-\left(F x_{2}\right)(t)=\lim _{\lambda \rightarrow \infty} \int_{0}^{t} U(t, \sigma) \lambda L_{\lambda}\left[f\left(\sigma, x_{1}(\sigma), \int_{0}^{\sigma} g\left(\sigma, \alpha, x_{1}(\alpha)\right) d \alpha\right)\right. \\
\left.-f\left(\sigma, x_{2}(\sigma), \int_{0}^{\sigma} g\left(\sigma, \alpha, x_{2}(\alpha)\right) d \alpha\right)+B(\sigma)\left(u_{1}(\sigma)-u_{2}(\sigma)\right)\right] d \sigma .
\end{gathered}
$$

For simplicity, we put $M:=\sup _{(t, s) \in \Delta_{T}} U(t, s)$. Then using assumptions (H7), (H8) and (H9) we obtain

$$
\begin{aligned}
\left\|\left(F x_{1}\right)(t)-\left(F x_{2}\right)(t)\right\| \leq & \gamma M \int_{0}^{t} \| f\left(\sigma, x_{1}(\sigma), \int_{0}^{\sigma} g\left(\sigma, \alpha, x_{1}(\alpha)\right) d \alpha\right) \\
& -f\left(\sigma, x_{2}(\sigma), \int_{0}^{\sigma} g\left(\sigma, \alpha, x_{2}(\alpha)\right) d \alpha\right) \| d \sigma \\
& +\gamma M\|B(\cdot)\|\left\|W^{-1}\right\| \gamma M
\end{aligned}
$$




$$
\begin{aligned}
& \times \int_{0}^{T} \| f\left(\alpha, x_{1}(\alpha), \int_{0}^{\alpha} g\left(\alpha, \tau, x_{1}(\tau)\right) d \tau\right) \\
& -f\left(\alpha, x_{2}(\alpha), \int_{0}^{\alpha} g\left(\alpha, \tau, x_{2}(\tau)\right) d \tau\right) d \alpha \| \\
\leq & \gamma M \int_{0}^{t} \ell_{f}\left(\left\|x_{1}(\sigma)-x_{2}(\sigma)\right\|\right. \\
& \left.+\| \int_{0}^{\sigma} g\left(\sigma, \tau, x_{1}(\tau)\right)-g\left(\sigma, \tau, x_{2}(\alpha)\right) d \tau\right) \| d \sigma \\
& +\gamma M\|B(\cdot)\|\left\|W^{-1}\right\| \gamma M \int_{0}^{T} \ell_{f}\left(\left\|x_{1}(\alpha)-x_{2}(\alpha)\right\|\right. \\
& \left.+\| \int_{0}^{\alpha} g\left(\alpha, \tau, x_{1}(\tau)\right)-g\left(\alpha, \tau, x_{2}(\alpha)\right) d \tau\right) \| d \alpha \\
\leq & \gamma M T \ell_{f}\left(\left\|x_{1}(\cdot)-x_{2}(\cdot)\right\|+T \ell_{g}\left\|x_{1}(\cdot)-x_{2}(\cdot)\right\|\right) \\
& +\gamma M\|B(\cdot)\|\left\|W^{-1}\right\| \gamma M T \ell_{f}\left(\left\|x_{1}(\cdot)-x_{2}(\cdot)\right\|\right) \\
& +\gamma M\|B(\cdot)\|\left\|W^{-1}\right\| \gamma M T \ell_{f}\left(T \ell_{g}\left\|x_{1}(\cdot)-x_{2}(\cdot)\right\|\right) \\
\leq & \gamma M T \ell_{f}\left(1+T \ell_{g}\right)\left\|x_{1}(\cdot)-x_{2}(\cdot)\right\| \\
& +\gamma^{2} M^{2} k_{1} k_{2} \ell_{f} T\left(1+T \ell_{g}\right)\left\|x_{1}(\cdot)-x_{2}(\cdot)\right\| \\
= & \left(1+T \ell_{g}\right)\left(\gamma M T \ell_{f}+\gamma^{2} M^{2} k_{1} k_{2} \ell_{f} T\right)\left\|x_{1}(\cdot)-x_{2}(\cdot)\right\| .
\end{aligned}
$$

Since, by hypotheses, $\left(1+T \ell_{g}\right)\left(\gamma M T \ell_{f}+\gamma^{2} M^{2} k_{1} k_{2} \ell_{f} T\right)<1$, then $F$ is a contraction mapping.

We are now ready to state the main result of this section.

Theorem 2. Assume that the hypotheses (H1)-(H9) are satisfied and suppose that $\left(1+T \ell_{g}\right)\left(\gamma M T \ell_{f}+\gamma^{2} M^{2} k_{1} k_{2} \ell_{f} T\right)<1$ for some $T>0$. Then the boundary integrodifferential control system (3.1) is exactly controllable on the interval $[0, T]$.

Proof. Let $x_{0}, y \in X$. From the above proposition we obtain that the operator $F$ defined in (3.5) has a fixed point $x(\cdot)$ which is a mild solution of the system (3.1). Furthermore, for $x(\cdot)$ choose a control $u(\cdot)$ as in (3.6). Then one can see that $x(\cdot)$ satisfies $x(T)=y$, giving the exact controllability.

\section{ApPlicAtion}

To illustrate the previous general assumptions and abstract results we consider in this section the following controlled population equation. 


$$
\left\{\begin{array}{l}
\frac{\partial}{\partial t} v(t, a)=-\frac{\partial}{\partial a} v(t, a)-\mu(t, a) v(t, a), \quad t \in[0, T], a \geq 0, \\
v(t, 0)=\int_{0}^{t} \int_{0}^{\infty} K\left(t, \tau, v(t, a) d a d \tau+\int_{0}^{\infty} \beta(t, a, v(t, a)) d a+b(t) u(t),\right. \\
u(0, a)=\varphi(a), \quad a \geq 0 .
\end{array}\right.
$$

Here the function $v(t, a)$ represents the density of individuals of the population of age $a$ at time $t$. The functions $\mu$ and $\beta$ correspond to the aging and the birth rates, respectively. We note that this equation is a special case of the general population equation investigated in [5].

We impose the following conditions:

(i) $\mu \in C^{1}\left([0, T], L^{\infty}\left(\mathbb{R}_{+}\right)\right)$and there exists a constant $\eta>0$ such that $\mu(t, a)>$ $\eta$ for all $t \geq 0$ and a.e. $a \in \mathbb{R}_{+}$.

(ii) $\beta$ is a positive and continuous function defined on $[0, T] \times \mathbb{R}_{+} \times \mathbb{R}$. Moreover, there exists a constant $\alpha>0$ such that

$$
|\beta(t, a, x)-\beta(t, a, \bar{x})| \leq \alpha|x-\bar{x}|
$$

for all $t \in[0, T] ; x, \bar{x} \in \mathbb{R}$ and a.e. $a \in \mathbb{R}_{+}$.

(iii) $b(\cdot) \in L^{2}(0, T)$ and $\varphi \in L^{1}\left(\mathbb{R}_{+}\right)$.

(iv) $K$ is a continuous function from $\Delta_{T} \times \mathbb{R}$ into $\mathbb{R}$ and there exists a constant $k>0$ such that

$$
|K(t, \tau, x)-K(t, \tau, \bar{x})| \leq k|x-\bar{x}|
$$

for all $(t, \tau) \in \Delta_{T}$ and $x, \bar{x} \in \mathbb{R}$.

We first write the system (4.1) as a boundary integrodifferential controlled system of the form (3.1) satisfying the hypotheses (H1)-(H6). For this purpose, we define the Banach spaces $X:=L^{1}\left(\mathbb{R}^{+}\right), U=\partial X:=\mathbb{R}$ and $D:=W^{1,1}\left(\mathbb{R}^{+}\right)$.

For each $t \geq 0$ we define the operator $A_{\max }(t): X \rightarrow X$ by

$$
\left(A_{\max }(t) \varphi\right)(a)=-\frac{\partial}{\partial a} \varphi(a)-\mu(t, a) \varphi(a)
$$

with domain $D\left(A_{\max }(t)\right)=D$ equipped with the norm

$$
\|\varphi\|_{D}:=\|\varphi\|_{X}+\left\|\varphi^{\prime}\right\|_{X}
$$

and the operator $L(t): D \longrightarrow \partial X$ by

$$
L(t) \varphi=\varphi(0)
$$

for all $\varphi \in D$.

The functions $g$ and $f$ are given by

$$
\begin{gathered}
g: \Delta_{T} \times X \longrightarrow X, \\
g(t, \tau, \varphi)(a)=K(t, \tau, \varphi(a))
\end{gathered}
$$


for all $(t, \tau) \in \Delta_{T}$ and $\varphi \in X$;

$$
\begin{gathered}
f:[0, T] \times X \times X \longrightarrow \partial X \\
f\left(t, \varphi_{1}, \varphi_{2}\right)=\int_{0}^{\infty} \varphi_{2}(a) d a+\int_{0}^{\infty} \beta\left(t, a, \varphi_{1}(a)\right) d a
\end{gathered}
$$

for all $t \in[0, T]$ and $\varphi_{1}, \varphi_{2} \in X$.

Remark 3. Using the Fubini's theorem, one can see that

$$
f\left(t, \varphi, \int_{0}^{t} g(t, \tau, \varphi) d \tau\right)=\int_{0}^{t} \int_{0}^{\infty} K\left(t, \tau, \varphi(a) d a d \tau+\int_{0}^{\infty} \beta(t, a, \varphi(a)) d a\right.
$$

for all $\varphi \in X$.

The system (4.1) is then a concrete case of the abstract boundary integrodifferential system (3.1).

Let us now check the assumptions (H1)-(H6).

Verification of $(\mathrm{H} 1)$ : Set $\mu_{\infty}:=\sup _{t \in[0, T]}\|\mu(t, \cdot)\|_{\infty}$ and let $\varphi \in D$ be arbitrary.

From the definition of $\|\cdot\|_{D}$, we have

$$
\begin{aligned}
\|\varphi\|_{D} & =\int_{0}^{\infty}|\varphi(a)| d a+\int_{0}^{\infty}\left|\varphi^{\prime}(a)\right| d a \\
& \leq \int_{0}^{\infty}|\varphi(a)| d a+\int_{0}^{\infty}\left|\varphi^{\prime}(a)-\mu(t, a) \varphi(a)\right| d a+\int_{0}^{\infty}|\mu(t, a) \varphi(a)| d a \\
& \leq\|\varphi\|_{X}+\left\|A_{\max }(t) \varphi\right\|_{X}+\int_{0}^{+\infty}|\mu(t, a) \varphi(a)| d a \\
& \leq\left(1+\mu_{\infty}\right)\left(\|\varphi\|_{X}+\left\|A_{\max }(t) \varphi\right\|_{X}\right) .
\end{aligned}
$$

On the other hand,

$$
\begin{aligned}
\|\varphi\|_{X}+\left\|A_{\max }(t) \varphi\right\|_{X} & =\|\varphi\|_{X}+\left\|\varphi^{\prime}-\mu(t, \cdot) \varphi\right\|_{X} \\
& \leq\left(1+\mu_{\infty}\right)\|\varphi\|_{D} .
\end{aligned}
$$

This shows the assumption (H1) with $C_{1}=\left(1+\mu_{\infty}\right)^{-1}$ and $C_{2}=\left(1+\mu_{\infty}\right)$.

Verification of (H2): From (4.4) and the assumptions on $\mu$, we derive that the map $t \mapsto A_{\max }(t) \varphi$ is continuously differentiable for each fixed $\varphi \in D$.

Verification of (H3): Since $\varphi(0)=\int_{0}^{+\infty} \frac{\partial}{\partial a} \varphi(a) d a$ for all $\varphi \in D$, it follows that

$$
\begin{aligned}
|L(t) \varphi| & =|\varphi(0)| \\
& \leq \int_{0}^{+\infty}\left|\frac{\partial}{\partial a} \varphi(a)\right| d a \\
& \leq\|\varphi\|_{D} .
\end{aligned}
$$


This shows the boundedness of $L(t)$. To prove the surjectivity of $L(t)$, let $x \in \partial X$ be arbitrary. Define

$$
\varphi(a):=e^{-a} x \quad \text { for all } a \in \mathbb{R}_{+} .
$$

We have $\varphi \in D$. Furthermore, one can easily see that $L(t) \varphi=x$ and therefore $L(t)$ is surjective.

Verification of (H4): From (4.5), we see that $L(t)$ is independent of $t$. Hence the mapping from $[0, T] \rightarrow \partial X, t \mapsto L(t) \varphi$ is continuously differentiable for each fixed $\varphi$ in $D$.

Verification of (H5): Let $\lambda>-\eta$, and $\varphi \in \operatorname{ker}\left(\lambda-A_{\max }(t)\right)$, i.e,

$$
\lambda \varphi(\cdot)+\frac{\partial}{\partial a} \varphi(\cdot)+\mu(t, \cdot) \varphi(\cdot)=0 .
$$

We have

$$
\begin{aligned}
|L(t) \varphi| & =|\varphi(0)| \\
& =\int_{0}^{+\infty}\left|\frac{\partial}{\partial a} \varphi(a)\right| d a \\
& =\int_{0}^{+\infty}(\lambda+\mu(t, a))|\varphi(a)| d a . \\
& \geq(\lambda+\eta)|\varphi|_{X} .
\end{aligned}
$$

Then (H5) is satisfied with $\omega=-\eta$ and $\gamma=1$.

Verification of (H6): Let $\lambda>0$. One can show that the resolvent operator of $A(t):=\left.A_{\max }(t)\right|_{k e r L(t)}$ is given by

$$
R(\lambda, A(t)) \varphi=\int_{0}^{\cdot} e^{-\int_{\tau}^{\cdot} \lambda+\mu(t, \sigma) d \sigma} \varphi(\tau) d \tau \quad \forall \varphi \in X .
$$

Then for $\lambda>-\eta$ we have

$$
\begin{aligned}
\|R(\lambda, A(t)) \varphi\| & =\int_{0}^{+\infty}|(R(\lambda, A(t)) \varphi)(a)| d a \\
& =\int_{0}^{+\infty}\left|\int_{0}^{a} e^{-\int_{\tau}^{a} \lambda+\mu(t, \sigma) d \sigma} \varphi(\tau) d \tau\right| d a \\
& \leq \int_{0}^{\infty} \int_{0}^{a} e^{-\int_{\tau}^{a} \lambda+\mu(t, \sigma) d \sigma}|\varphi(\tau)| d \tau d a \\
& =\int_{0}^{+\infty}|\varphi(\tau)| \int_{\tau}^{+\infty} e^{-\int_{\tau}^{a} \lambda+\mu(t, \sigma) d \sigma} d a d \tau \\
& \leq \int_{0}^{+\infty}|\varphi(\tau)| \int_{\tau}^{+\infty} e^{-\int_{\tau}^{a}(\lambda+\eta) d \sigma} d a d \tau \\
& \leq \frac{1}{\lambda+\eta} \int_{0}^{+\infty}|\varphi(\tau)| d \tau
\end{aligned}
$$




$$
=\frac{1}{\lambda+\eta}\|\varphi\| \text {. }
$$

Hence, we obtain that $(A(t))_{t \in[0, T]}$ is stable with stability constants $M=1$ and $\omega=-\eta$.

We conclude this section by the following controllability result of the controlled population equation (4.1).

Proposition 2. Under the above assumptions and if we assume that

$$
(1+T k)\left(T(1+\alpha)+\|b(\cdot)\|\left\|W^{-1}\right\|(1+\alpha) T\right)<1,
$$

then the controlled population problem (4.1) is exactly controllable.

Proof. From (4.7) and (4.2) one can verify that the function $f$ satisfies (H7) with constant $(1+\alpha)$. Using (4.6) and (4.3), one can also verify that the function $g$ satisfies (H8) with constant $k$. The result is then a direct application of Theorem 2.

\section{REFERENCES}

[1] K. Balachandran and R. Sakthivel, "Controllability of integrodifferential systems in Banach spaces," Appl. Math. Comp., vol. 118, pp. 63-71, 2001.

[2] S. Boulite, L. Maniar, and M. Moussi, "Wellposedness and asymptotic behaviour of nonautonomous boundary cauchy problems," Forum Math., vol. 18, pp. 611-638, 2006.

[3] J. C. Chang, "Solutions to non-autonomous integrodifferential equations with infinite delay," $J$. Math. Anal. Appl., vol. 331, pp. 137-151, 2007.

[4] J. C. Chang and C. L. Lang, "Local existence for nonlinear Volterra integrodifferential equations with infinite delay," Nonlinear Anal., Theory Methods Appl., vol. 68, pp. 2943-2956, 2008.

[5] J. C. Chang and S. Y. Shaw, "Solutions to boundary cauchy problems with infinite delay," Nonlinear Anal., Theory Methods Appl., vol. 73, pp. 1670-1683, 2010.

[6] H. Ding, T. Xiao, and J. Liang, "Asymptotically almost automorphic solutions for some integrodifferential equations with nonlocal initial conditions," J. Math. Anal. Appl., vol. 338, pp. 141-151, 2008.

[7] K. Ezzinbi and S. Ghnimi, "Existence and regularity of solutions for neutral partial functional integrodifferential equations," Nonlinear Anal., Real World Appl., vol. 11, pp. 2335-2344, 2010.

[8] W. Fitzgibbon, "Semilinear integrodifferential equations in Banach space," Nonlinear Anal., Theory Methods Appl., vol. 4, pp. 745-760, 1980.

[9] G. Greiner, "Perturbing the boundary conditions of a generator," Houston J. Mathematics, vol. 13, pp. 213-229, 1987.

[10] M. Heard, "An abstract semilinear hyperbolic Volterra integrodifferential equation," J. Math. Anal. Appl., vol. 80, pp. 175-202, 1981.

[11] E. Hernández M, J. Paulo C, and D. Santos, "Asymptotically almost periodic and almost periodic solutions for a class of partial integrodifferential equations," Electon. J. of Differ. Equ., vol. 2006, pp. 1-8, 2006.

[12] A. Jawahdou, "Mild solutions of functional semilinear evolution Volterra integrodifferential equations on an unbounded interval," Nonlinear Anal., Theory Methods Appl., vol. 74, pp. 7325-7332, 2011.

[13] H. Kellermann, "Linear evolution equations with time-dependent domain," Semesterbericht Funktionalanalysis, Tübingen, vol. Wintersemester, pp. 15-45, 1985. 
[14] J. Liang and T. J. Xiao, "Semilinear integrodifferential equations with nonlocal initial conditions," Comput. Math. Appl., vol. 47, pp. 863-875, 2004.

[15] M. Meehan and D. O'Regan, "Existence theory for nonlinear Volterra integrodifferential and integral equations," Nonlinear Anal., Theory Methodes Appl., vol. 31, pp. 317-341, 1998.

[16] G. S. Muldownfy, "Bounds for solutions of nonlinear integrodifferential equations," J. Math. Anal. Appl., vol. 23, pp. 487-499, 1968.

[17] R. Nagel and E. Sinestrari, "Nonlinear hyperbolic Volterra integrodifferential equations," Nonlinear Anal., Theory Methods Appl., vol. 27, pp. 167-168, 1996.

[18] T. L. Nguyen, "On nonautonomous functional-differential equations," J. Math. Anal. Appl., vol. 239, pp. 158-174, 1999.

[19] M. D. Quinn and N. Carmichael, "An approach to nonlinear control problems using the fixed point methods, degree theory and pseudo-inverses," Numer. Funct. Anal. Optim., vol. 7, pp. 197-219, 1984-1985.

[20] S. Ruan, "Asymptotic stability for Volterra integrodifferential systems," Appl. Math. Comput., vol. 52, pp. 207-222, 1992.

[21] V. S. Sergeev, "Stability of solutions of Volterra integrodifferential equations," Math. Comput. Model., vol. 45, pp. 1376-1394, 2007.

[22] Q. Tiehu, "Asymptotic behavior of a class of abstract semilinear integrodifferential equations and applications," J. Math. Anal. Appl., vol. 233, pp. 130-147, 1999.

[23] Z. Yan, "On solutions of semilinear evolution integrodifferential equations with nonlocal conditions," Tamkang J. Math., vol. 40, pp. 257-269, 2009.

\section{Author's address}

\section{Moussi}

University Mohamed I, Faculty of Sciences, Department of Mathematics and Informatics, BV Mohamed VI, 60000 Oujda, Morocco

E-mail address: mohmoussi@hotmail.com 